\title{
VISTA.COMP - an engineered checkpoint receptor agonist that potently suppresses T cell-mediated immune responses
}

Aaron Prodeus, ${ }^{1,2}$ Aws Abdul-Wahid, ${ }^{2}$ Amanda Sparkes, ${ }^{2}$ Nicholas W. Fischer, ${ }^{1,2}$ Marzena Cydzik, ${ }^{2}$ Nicholas Chiang, ${ }^{2,3}$ Mays Alwash, ${ }^{2,3}$ Alessandra Ferzoco, ${ }^{2,4}$ Nathalie Vacaresse, ${ }^{2}$ Michael Julius, ${ }^{1,2,4}$ Reginald M. Gorczysnki, ${ }^{3,5}$ and Jean Gariépy ${ }^{1,2,3}$

'Department of Medical Biophysics, University of Toronto, Toronto, Ontario, Canada. ${ }^{2}$ Sunnybrook Research Institute, Toronto, Ontario, Canada. ${ }^{3}$ Department of Pharmaceutical Sciences and ${ }^{4}$ Department of Immunology, University of Toronto, Toronto, Ontario, Canada. ${ }^{5}$ Transplant Research Division, University Health Network, Toronto, Ontario, Canada

$\underline{V}$-domain immunoglobulin suppressor of $\underline{T}$ cell activation (VISTA) is a recently discovered immune checkpoint ligand that functions to suppress $T$ cell activity. The therapeutic potential of activating this immune checkpoint pathway to reduce inflammatory responses remains untapped, largely due to the inability to derive agonists targeting its unknown receptor. A dimeric construct of the IgV domain of VISTA (VISTA-Fc) was shown to suppress the activation of T cells in vitro. However, this effect required its immobilization on a solid surface, suggesting that VISTA-Fc may display limited efficacy as a VISTA-receptor agonist in vivo. Herein, we have designed a stable pentameric VISTA construct (VISTA.COMP) by genetically fusing its IgV domain to the pentamerization domain from the cartilage oligomeric matrix protein (COMP). In contrast to VISTA-Fc, VISTA.COMP does not require immobilization to inhibit the proliferation of $\mathrm{CD} 4^{+} \mathrm{T}$ cells undergoing polyclonal activation. Furthermore, we show that VISTA.COMP, but not VISTA-Fc, functions as an immunosuppressive agonist in vivo capable of prolonging the survival of skin allografts in a mouse transplant model as well as rescuing mice from acute concanavalin-A-induced hepatitis. Collectively, we believe our data demonstrate that VISTA.COMP is a checkpoint receptor agonist and the first agent to our knowledge targeting the putative VISTA-receptor to suppress $\mathrm{T}$ cell-mediated immune responses.

Conflict of interest: The authors have declared that no conflict of interest exists.

Submitted: March 29, 2017 Accepted: August 10, 2017 Published: September 21, 2017

Reference information: JCI Insight. 2017;2(18):e94308. https://doi.org/10.1172/jici. insight.94308.

\section{Introduction}

$\mathrm{T}$ cell activity is tightly regulated by a spectrum of costimulatory and coinhibitory signals, allowing for protection against invading pathogens or malignant cells, while maintaining self-tolerance. To date, several negative checkpoint receptors, such as PD-1 and CTLA-4, have been identified that function to suppress T cell activity (1-4). Antibody-mediated blockade of these pathways has been shown to promote remarkable antitumor immune responses $(3,5,6)$. Conversely, ligands that activate these immunoinhibitory pathways regulate the progression of uncontrolled immune responses in T cell-mediated autoimmune and inflammatory disorders (7-9)

$\underline{V}$-domain immunoglobulin suppressor of $\underline{T}$ cell activation (VISTA, also known as PD-1H, Dies1, $\mathrm{DD} 1 \alpha, \mathrm{Gi24})$ is a newly characterized checkpoint ligand, expressed primarily on CD11b ${ }^{\text {hi }}$ myeloid cells, which negatively regulates $\mathrm{T}$ cell responses upon binding to an as-of-yet undiscovered cell surface receptor (VISTA-receptor) $(10,11)$. VISTA is also expressed on naive $\mathrm{CD}^{+}$and $\mathrm{CD} 8^{+} \mathrm{T}$ cells, where it negatively regulates $\mathrm{T}$ cell responses, suggesting a dual role of VISTA as both a checkpoint ligand and receptor (12). Structurally, VISTA shares substantial homology with PD-1 and PD-L1, consisting of an N-terminal IgV domain followed by a single-membrane spanning domain and cytoplasmic tail. As in the case of the PD-1/ PD-L1 pathway, blockade of VISTA using monoclonal antibodies has been demonstrated to provoke antitumor immune responses in several mouse models, suggesting a role for VISTA/VISTA-receptor signaling in the promotion of tumor immune evasion $(13,14)$.

VISTA has also been implicated in regulating the progression of autoimmune diseases. It has been reported that VISTA-deficient $\left(\right.$ VISTA $^{--}$) mice bred on a lupus-prone background develop accelerated and severe systemic lupus erythematosus (15). Similarly, VISTA ${ }^{-/-}$2D2 T cell receptor-transgenic mice exhibit 
increased levels of peripheral encephalitogenic T cells and develop an exacerbated form of experimental autoimmune encephalomyelitis (16). More recently, it was shown that VISTA-deficient mice exhibited worsened disease in a model of imiquimod-induced psoriasis, where enhanced TLR7 signaling in DCs led to augmented IL-23 production and increased expression of IL-17A in T cells (17). The VISTA ${ }^{-/}$mice bred on a wild-type C57BL/6 background display a mild proinflammatory phenotype, exemplified by an increase in DCs and a rise in T cell activation markers, but were not reported to develop inflammatory disorders (16). In slight contrast, another study described a more severe phenotype, where VISTA ${ }^{-/}$C57BL/6 mice developed glomerulonephritis at 10 months of age (18). Taken together, these studies indicate a potential therapeutic advantage in promoting VISTA-mediated immunosuppression to treat autoimmune or inflammatory diseases.

While agonistic anti-VISTA antibodies have been reported (19), the derivation of novel agonists aimed at the putative VISTA-receptor remains challenging due to its unknown identity. A dimeric version of VISTA (VISTA-Fc) has been previously reported to suppress both mouse and human $\mathrm{T}$ cell activation in vitro. However, this activity requires the immobilization of this ligand on a solid surface (10, 13). Accordingly, no study has reported that a soluble form of the VISTA extracellular domain can act as an effective agonist to suppress inflammatory responses in vivo. In this study, we describe the engineering of a stable pentameric form of the VISTA extracellular domain (VISTA.COMP), which in contrast to VISTA-Fc effectively inhibits $\mathrm{CD}^{+} \mathrm{T}$ cell activation as a soluble ligand. Moreover, we present evidence that this VISTA-receptor agonist suppresses inflammatory responses in vivo, in the context of prolonging murine skin allograft survival and in protecting mice from lethal acute hepatitis. The results described provide support for continued characterization of VISTA-receptor agonists (such as VISTA.COMP) as a therapeutic strategy to treat autoimmune and inflammatory disorders.

\section{Results}

A recombinant pentameric VISTA-IgV construct suppresses $T$ cell activation as a soluble ligand in vitro. Consistent with previous reports $(10,11)$, we found that dimeric VISTA-Fc constructed by fusing the VISTA-IgV domain with the $\mathrm{Fc}_{\mathrm{C}}$ region from $\mathrm{IgG} 1$, only suppressed the proliferation of anti-CD3 stimulated $\mathrm{CD} 4^{+} \mathrm{T}$ cells when immobilized on a culture dish (Figure 1A). This finding suggests that its use in vivo to suppress $\mathrm{T}$ cell activity may be limited due to its inability to successfully agonize the putative VISTA-receptor. We hypothesized that the lack of activity of soluble VISTA-Fc in vitro may reflect insufficient avidity toward its receptor or a lack of ability to cluster the VISTA-receptor on the cell surface. Consequently, we engineered a higher-order VISTA oligomer to address these challenges, with a view to generate an agonist that can effectively suppress T cell stimulation both in vitro and in vivo. Previous studies have reported on the use of the short pentamerization domain from the cartilage oligomeric matrix protein (COMP) to construct stable recombinant pentamers with increased avidity and activity relative to lesser-order multimers (20). The COMP pentamerization domain is a short 44-amino acid sequence that spontaneously assembles into a bundle of $5 \alpha$-helices arranged in a parallel orientation and is stabilized by disulphide bridges (21). Previously, the proangiogenic factor angiopoietin 1, when fused to the COMP domain (COMP-Ang1), showed increased stability relative to native Ang1, leading to an increased induction of angiogenesis in vivo (20). Thus, a recombinant VISTA pentamer (VISTA.COMP) was constructed by genetically fusing the VISTA $\mathrm{IgV}$ domain to the COMP pentamerization domain. Recombinant VISTA.COMP was produced in a mammalian expression system, yielding a pentameric protein of approximately $250 \mathrm{kDa}$ (Figure $1 \mathrm{~B}$ ).

In contrast to VISTA-Fc, soluble VISTA.COMP substantially suppressed the expansion and proliferation of isolated anti-CD3 stimulated $\mathrm{CD}^{+} \mathrm{T}$ cells (Figure $1 \mathrm{C}$ ). The recombinant COMP domain alone showed a negligible effect on $\mathrm{T}$ cell expansion and proliferation, suggesting that this activity is due to VISTA signaling and not off-target events associated with the COMP domain. In addition, soluble VISTA.COMP significantly diminished $(P<0.005)$ the secretion of inflammatory cytokines IL-2 (Figure 1D) and IFN- $\gamma$ (Figure 1E) by stimulated $\mathrm{CD}^{+} \mathrm{T}$ cells. The efficacy of VISTA.COMP suppression was inversely correlated with the strength of TCR stimulation, as increased anti-CD3 stimulation led to increases in T cell division in the presence of VISTA.COMP (Supplemental Figure 1A; supplemental material available online with this article; https://doi. org/10.1172/jci.insight.94308DS1). In addition to its ability to suppress $\mathrm{T}$ cell proliferation in response to a polyclonal stimulus, VISTA.COMP readily suppressed the induction of cytotoxic T lymphocytes (CTLs), in a dose-dependent manner, in allogenic mixed-leukocyte cultures (Supplemental Figure 1B). These results demonstrate that this VISTA pentamer represents an effective agonist, capable of activating the VISTA-receptor on T 
A

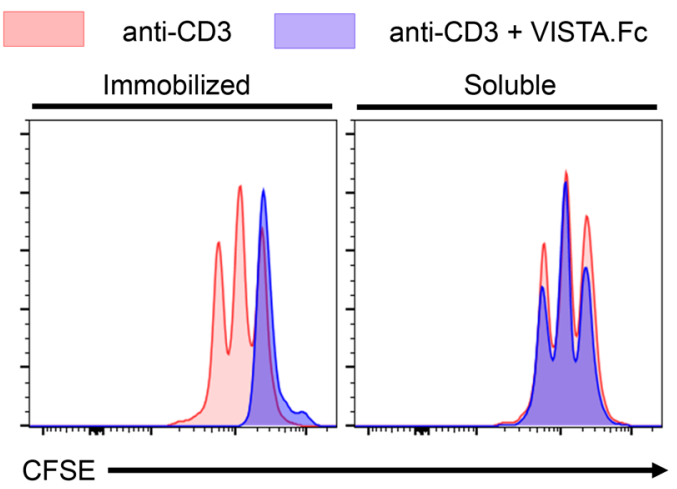

C
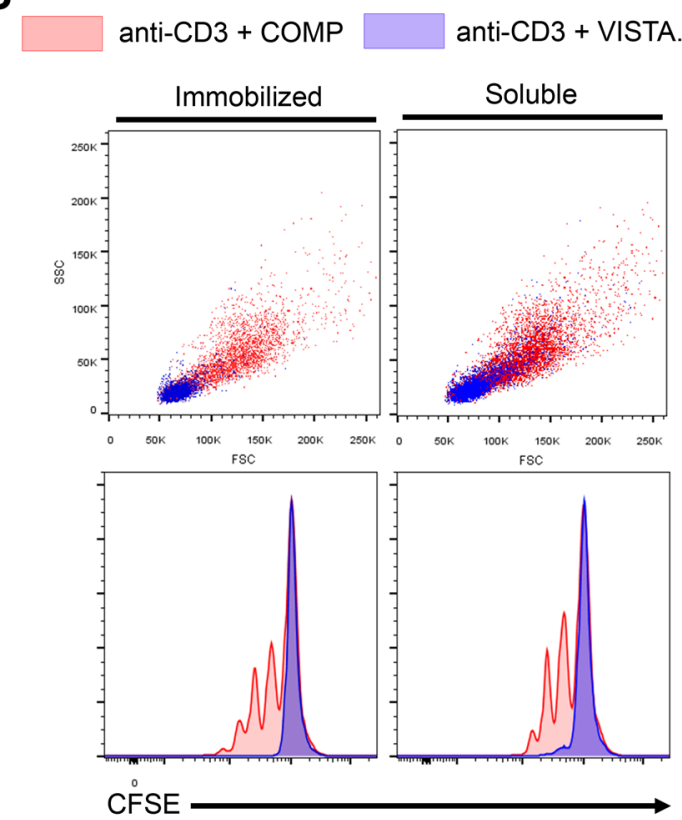

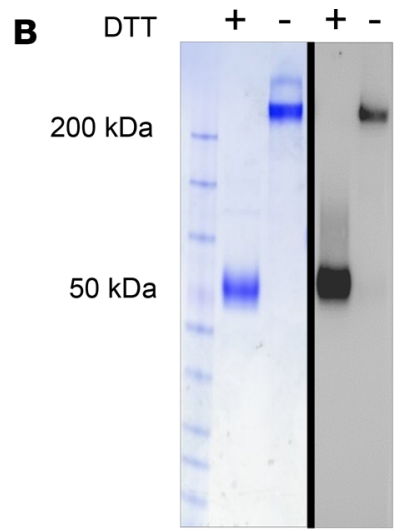

D
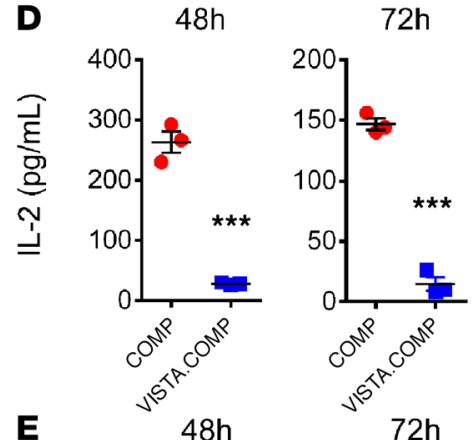

$72 \mathrm{~h}$

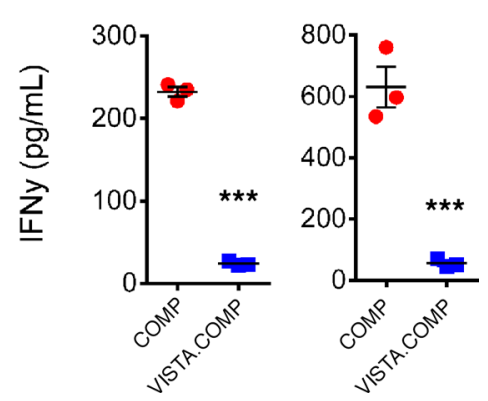

Figure 1. Pentameric VISTA.COMP suppresses $\mathrm{T}$ cell activation and proliferation as a soluble ligand in vitro. (A) CFSE-labeled purified murine CD4 ${ }^{+} \mathrm{T}$ cells were activated by plate-bound anti-CD3 antibody $(2.5 \mu \mathrm{g} / \mathrm{ml}$ ) in the presence (blue) or absence (red) of immobilized (left) or soluble (right) VISTA-Fc $(10 \mu \mathrm{g} / \mathrm{ml})$ for 48 hours. VISTA-Fc suppresses the proliferation of CD4+ T cells when immobilized but not when added as a soluble ligand in the culture media. (B) Recombinant VISTA.COMP was expressed as described in the Methods, and the purity and pentameric status was confirmed by SDS-PAGE and Western blot in the presence or absence of a reducing agent (DTT). Reduced VISTA.COMP migrates as a single band approximately $50 \mathrm{kDa}$, while the disulfide-stabilized pentamer has an apparent mass of $250 \mathrm{kDa}$. (C) Proliferation assay of CD4+ $\mathrm{T}$ cells undergoing activation in the presence of coated ( 9 $\mu \mathrm{g} / \mathrm{ml}$, left) or soluble ( $12 \mu \mathrm{g} / \mathrm{ml}$, right) VISTA.COMP (blue) or COMP (red). Soluble VISTA.COMP suppresses T cell expansion (top, forward scatter [FSC] and side scatter [SSC] profiles) and proliferation (bottom, CFSE dilution). Culture medium was harvested from CD4 ${ }^{+}$T cells 48 and 72 hours after anti-CD activation in the presence of COMP or VISTA.COMP $(10 \mu \mathrm{g} / \mathrm{ml})$, and IL-2 (D) and IFN- $\gamma(\mathbf{E})$ secretion was quantified by ELISA. In addition to proliferation, VISTA.COMP was found to significantly suppress IL-2 and IFN- $\gamma$ secretion from T cells (data represent mean \pm SEM, ${ }^{* * *} P<0.005$ relative to COMP control by Student's $t$ test, $n=3$ ). All data shown are representative of at least 3 independent experiments.

cells to regulate their activity without requiring immobilization on a solid surface, as is the case with VISTA-Fc.

VISTA.COMP binds to and suppresses activation of a clonal $T$ cell line. In addition to primary $\mathrm{CD} 4^{+} \mathrm{T}$ cells, we found that a CD4- murine IL-2-dependent T cell clone (2.10) was sensitive to VISTA inhibitory signaling, providing a controlled system to assay the function of VISTA-receptor agonists (22). Consistent with what was observed in primary $\mathrm{CD} 4^{+} \mathrm{T}$ cells, VISTA-Fc suppressed anti-CD3-induced proliferation only when immobilized on a solid surface, while VISTA.COMP completely suppressed proliferation when both immobilized and soluble in culture medium $(P<0.01)$ (Figure 2A). Titration of soluble VISTA.COMP and VISTA-Fc demonstrated that VISTA.COMP suppressed anti-CD3-induced 2.10 cell proliferation at concentrations as low as $1 \mu \mathrm{g} / \mathrm{ml}(P<0.01)$, meanwhile VISTA-Fc had no detectable activity at concentrations as high as $30 \mu \mathrm{g} / \mathrm{ml}$ (Figure $2 \mathrm{~B}$ ). In addition to suppressing proliferation, intracellular flow cytometry 
A

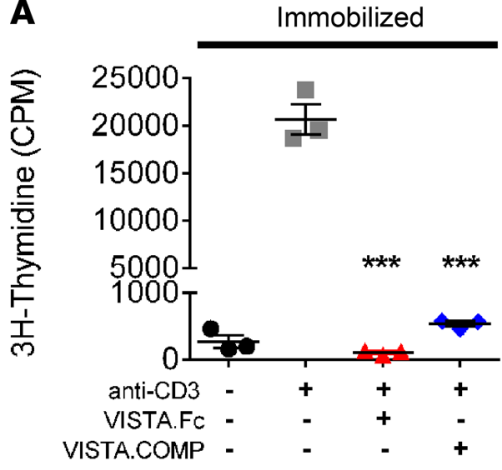

C
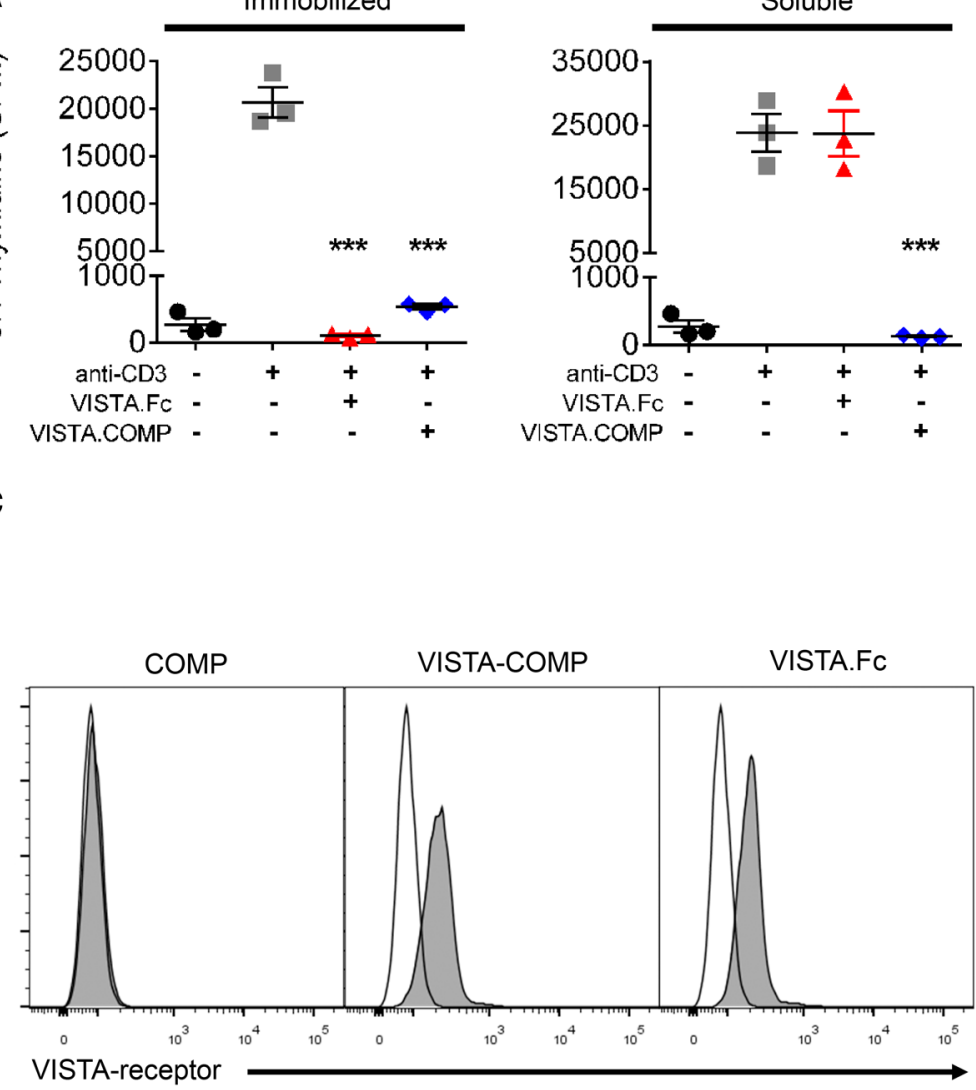

B



Concentration $(\mu \mathrm{g} / \mathrm{mL})$

D

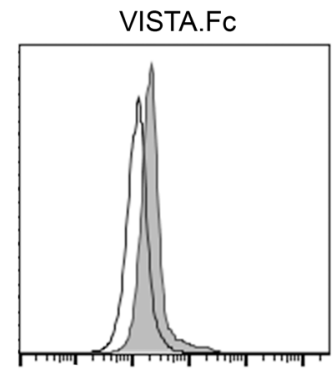

VISTA.FC + VISTA.COMP

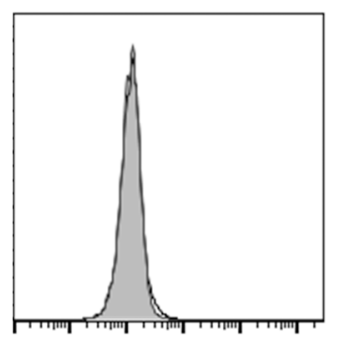

PE anti-Ig

Figure 2. VISTA.COMP binds to a clonal T cell line and suppresses its activation. (A) 2.10 clonal T cells were activated with immobilized anti-CD3 antibody $(3 \mu \mathrm{g} / \mathrm{ml})$ in the presence of immobilized or soluble VISTA-Fc or VISTA.COMP $(10 \mu \mathrm{g} / \mathrm{ml})$, and proliferation was measured by pulsing cells with ${ }^{3} \mathrm{H}$-thymidine in the last 6 hours of a 24-hour culture. As observed with primary CD4 ${ }^{+} T$ cells, pentameric VISTA.COMP suppresses proliferation when immobilized or when added soluble in culture media, whereas VISTA-Fc only exhibits suppressive activity when immobilized (data represent mean $\pm \mathrm{SEM}$, ${ }^{* * *} P<0.01$ relative to anti-CD3 stimulated control by Student's $t$ test, $n=3$ ). Data are representative of at least three independent experiments. (B) Titration of soluble VISTA. COMP (blue) or VISTA-Fc (red) on 2.10 cells activated as described in A. Data show a lack of an antiproliferative effect on cells by soluble VISTA-Fc at high concentrations (each point represents mean \pm SEM). (C) FACS analysis of biotinylated COMP, biotinylated VISTA.COMP, and VISTA-FC (shaded histogram) binding to 2.10 clonal T cells compared with unstained control (empty histograms). Data are representative of 3 independent experiments. (D) FACS-derived histograms of VISTA-Fc binding to 2.10 cells (shaded histograms) in the presence or absence of equimolar unlabeled VISTA.COMP. Empty histograms represent unstained cells. VISTA.COMP readily out competes VISTA-Fc binding to these cells.

shows that soluble VISTA.COMP, but not VISTA-Fc, suppressed stimulated 2.10 cell IL-2 secretion within 4 hours of exposure $(P<0.05)$, suggesting an immediate and rapid effect of VISTA.COMP (Supplemental Figure 2). Mechanistically, these results are consistent with the previous finding that exposing naive $\mathrm{CD} 4^{+}$ $\mathrm{T}$ cells to immobilized VISTA-Fc led to long-term suppression of T cells upon transfer to anti-CD3-coated wells (in the absence of further VISTA-Fc), suggesting a role for VISTA signaling as a critical early regulator of $\mathrm{T}$ cell activation (11). Flow cytometry was then performed on the 2.10 cell line using VISTA-Fc, COMP, or VISTA.COMP to determine if the inability of soluble VISTA-Fc to bind to the VISTA-receptor on $\mathrm{T}$ cells contributes to the lack of suppressive activity. VISTA.COMP and COMP were labeled with an equivalent number of biotins groups, and cell-bound biotinylated proteins were detected with PE-streptavidin, while bound VISTA-Fc was detected with PE-anti-IgG. Both VISTA-Fc and VISTA.COMP were found to bind to naive $2.10 \mathrm{~T}$ cells, while the baseline signal observed for COMP confirmed the absence of nonspecific binding arising from the pentamerization domain alone (Figure 2C). Unlike VISTA.COMP, the VISTA-Fc signal, however, could be readily displaced by additional washing steps, suggesting that its 
A

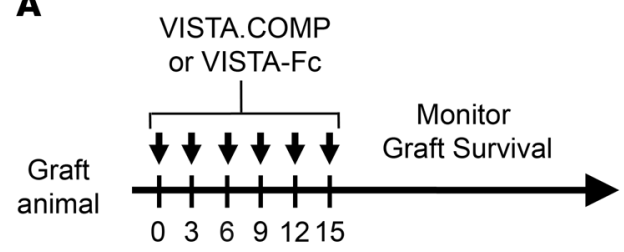

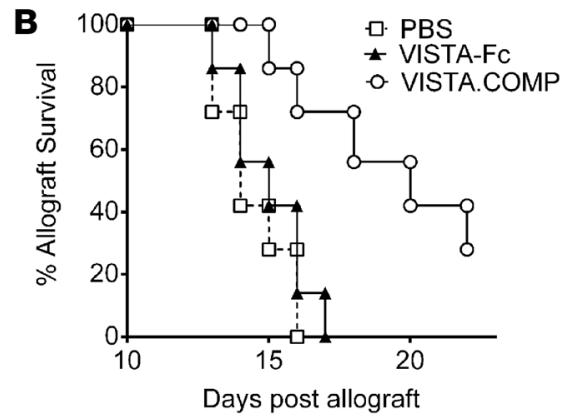

D

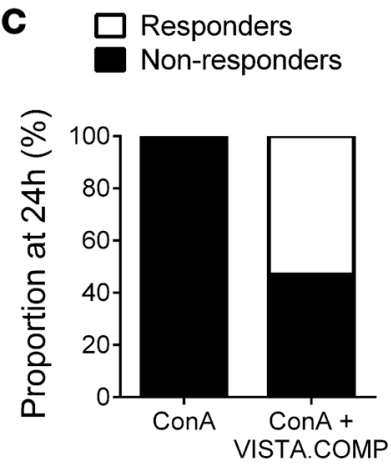



$\mathbf{E}$

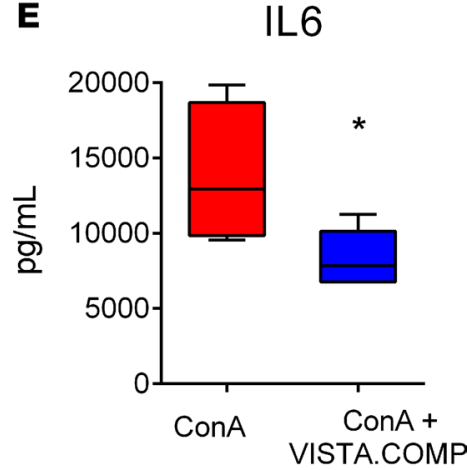

Figure 3. VISTA.COMP suppresses immune responses in vivo. (A) Schematic representation of the skin allograft rejection model. On day $0, B A L B / C$ animals are engrafted with skin from $C 57 \mathrm{BL} / 6$ mice and subsequently treated with VISTA. COMP of VISTA-Fc or PBS over the course of 15 days (arrows). Graft survival was monitored daily by a blinded investigator. (B) Treatment with VISTA.COMP, but not VISTA-Fc, significantly prolonged survival of skin allografts $(n=7, P<0.05$ by Mann-Whitney $U$ test). VISTA.COMP activity was confirmed in 2 independent experiments (pooled $n=11, P<0.01$ by Mann-Whitney $U$ test). (C) Treatment with VISTA.COMP 2 hours prior to ConA injection rescued C57BL/ 6 mice from lethal hepatic injury at 24 hours ( $n=13, P<0.05$ using proportion test). Moribund animals are classified as nonresponders (see Methods). Data are pooled from 3 independent trials. Treated mice exhibited a significant decrease in serum TNF- $\alpha(n=$ $5,{ }^{*} P<0.05$ by Student's $t$ test) (D) and IL- $6\left(n=5,{ }^{*} P<0.05\right.$ by Student's $t$ test) (E) 3 hours after ConA injection.

interaction with the putative VISTA-receptor is of low affinity (data not shown). Furthermore, in a competition assay, the high-avidity VISTA.COMP present at equimolar concentrations completely inhibited VISTA-Fc binding to 2.10 cells (Figure 2D). Altogether, these findings demonstrate that a soluble dimeric VISTA ligand is not sufficient to activate immunoinhibitory signaling through this pathway in vitro, while the high-avidity VISTA.COMP is capable of binding to stimulating the VISTA-receptor on T cells.

VISTA.COMP prolongs the survival of allografts in a MHC-mismatched skin transplant model. The ability of VISTA.COMP to suppress $\mathrm{T}$ cell activity in vitro as a soluble ligand suggests that it may be a useful agonist to suppress proinflammatory responses in vivo. To this end, VISTA.COMP was first tested in a murine skin allograft model. Here, BALB/C mice received nonhistocompatible skin allografts (from C57BL/6 donors) before receiving treatment with VISTA.COMP, VISTA-Fc, or a saline control combined with lowdose rapamycin (Figure 3A). We have demonstrated in the past that this dose of rapamycin has no effect on graft survival as a monotherapy (23). Strikingly, VISTA.COMP significantly prolonged the survival of skin allografts, with only 1 of 6 allografts rejected in the VISTA.COMP treatment group at the last day of treatment (day 15), relative to 6 of 6 rejected allografts observed in the saline control group $(P<0.05$, Mann-Whitney $U$ test) (Figure 3B). Importantly, and in support with our hypothesis, VISTA-Fc treatment did not prolong allograft survival.

VISTA.COMP dampens immune responses and reduced lethality in the ConA-induced hepatitis mouse model. The immunosuppressive effects of VISTA.COMP were also evaluated in an acute inflammatory hepatic model, namely concanavalin-A-induced (ConA-induced) hepatitis. Here, the administration of ConA induces acute liver inflammation mediated by a polyclonal activation of $\mathrm{CD} 4^{+} \mathrm{T}$ and NKT cells, thereby providing a useful model to assess the suppressive activity of VISTA.COMP on T cells in vivo (24). Notably, it has been previously shown that agonistic anti-VISTA antibodies directed toward VISTA on T cells 
can rescue mice from lethal ConA-induced hepatic injury (12). However, the usefulness of using a VISTA-receptor agonist has not been described. We found that prophylactic treatment of mice with VISTA. COMP rescued 7 of 13 (53\%) C57BL/ 6 mice from succumbing to a lethal dose of ConA (Figure 3C). This treatment correlated with a significant reduction of TNF- $\alpha$ and IL-6 levels in serum 3 hours after ConA injection $(P<0.05)$ (Figure 3, D and $\mathrm{E})$. As seen in the skin allograft model, VISTA-Fc treatment did not have a significant effect on survival or in reduction of serum TNF- $\alpha$ and IL-6 levels (Supplemental Figure 3 ). The striking results from these two acute inflammatory disease models suggest that VISTA.COMP may serve as a strong agonist to suppress inflammatory responses in vivo.

\section{Discussion}

The activation of immune checkpoint receptors on $\mathrm{T}$ cells is often initiated through the binding of an $\operatorname{IgV}$ domain displayed by a protein ligand, such as PD-L1, expressed on APCs to a complementary IgV domain of its cognate immune checkpoint receptor PD-1 on T cells. Past structural studies have demonstrated that monomeric forms of these IgV domains involving PD1/PD-L1 and CD28/CD80 or CD28/CD86 interact with each other with modest affinity, reflected by $\mathrm{K}_{\mathrm{d}}$ values typically in the low micromolar $(\mu \mathrm{M})$ range $(25,26)$. To activate checkpoint receptors on $\mathrm{T}$ cells in vitro, these immune checkpoint ligands are often expressed as oligomers, such as Fc fusion proteins, and are immobilized on a surface; a presentation that mimics avidity events taking place when such immune checkpoint domains are displayed on the surface of APCs and T cells. In line with this avidity requirement, there are currently no soluble agonists directed at VISTA, PD-1, or other checkpoint receptors in clinical trials (9).

Here, we have shown that the pentamerization of the VISTA-IgV domain by fusing it to a short, bundle-forming $\alpha$-helical peptide derived COMP generates a VISTA-IgV scaffold that is sufficient to agonize the putative VISTA-receptor to suppress $\mathrm{T}$ cells in vitro and dampen acute inflammatory reactions in models of MHC-mismatched skin transplants as well as acute inflammatory hepatic injury. Altogether, our data support a role for VISTA.COMP as a high-avidity checkpoint receptor agonist. Comparisons among immobilized and soluble VISTA-Fc and VISTA.COMP show that their ability to act as VISTA-receptor agonists depends on the level of oligomerization, favoring the higher-avidity multimer created by immobilization of the VISTA-IgV on a solid surface or by multimerization using the COMP pentamerization domain. We have found the COMP domain to be a useful scaffold for expressing stable VISTA pentamers. However, the affinity/avidity and mechanism of action of VISTA multimers on immune cells is currently difficult to characterize, largely due to the unknown identity of its receptor. Our data, and those of others, suggest that VISTA functions as an early regulator of $\mathrm{T}$ cell activation, with VISTA signaling capable of imprinting long-lasting suppressive effects on $\mathrm{T}$ cells $(10,11,27)$. Currently, the molecular pathways at play remain to be elucidated and may become clearer upon discovery of the putative receptor. Notably, it has been previously reported that VISTA interacts with itself in a homotypic fashion (18). However, we have not observed a direct homotypic interaction in binding assays using purified VISTA.COMP and VISTA-Fc. More importantly, the lack of support for a physiologically relevant VISTA-homotypic interaction between APC and T cells comes from a reported experiment in which OVA peptide-recognizing $\mathrm{T}$ cells isolated from $\mathrm{VISTA}^{-/-}$mice remain sensitive to suppression by VISTA expressed on the surface of APCs (12). We also found that the high-avidity VISTA.COMP did not bind to homologous checkpoint receptors such as PD-1 and PD-L1 (data not shown), confirming conclusions reached by others $(11,12)$. Thus, it seems apparent that VISTA functions as a checkpoint ligand through a putative counterreceptor expressed on T cells which has yet-to-be identified (27). More recently, a high-throughput screening using a single-pass transmembrane protein library has identified IGSF11 (also known as VSIG3) as a VISTA-binding partner (28). Experimentally, we have since confirmed that recombinant human IGSF11 does bind to recombinant human VISTA.COMP $\left(\mathrm{K}_{\mathrm{D}}=0.024 \mu \mathrm{M}\right)$ and VISTA-Fc $\left(\mathrm{K}_{\mathrm{D}}=1.3 \mu \mathrm{M}\right)$ using surface plasmon resonance binding assays (data not shown). Interestingly, IGSF11 is predominantly expressed in the brain and sex organs, and the physiological relevance of the VISTA-IGSF11 interaction is not yet understood (28). Current research is ongoing to determine if IGSF11 is expressed by myeloid, lymphoid, or cancer cells, with a view to characterize if an immunological role for this molecule exists. Research is currently ongoing to characterize the cell-signaling mechanisms triggered by VISTA.COMP in both mouse and human T cells. Nonetheless, our data, combined with the observation of exacerbated autoimmune diseases upon genetic deletion of VISTA (15-18), suggest a potential utility of targeting the VISTA-mediated immunosuppression pathway using a high-avidity VISTA-IgV containing scaffold to clinically suppress undesired immune responses. 


\section{Methods}

Recombinant protein expression and purification. VISTA-Fc was produced by cloning a synthetic dsDNA coding for the extracellular domain (residues 16-194) of murine VISTA upstream of the human IgG-1 Fc region (GeneArt; Thermo Fisher Scientific) into the pcDNA-3.4 expression plasmid (Thermo Fisher Scientific). The plasmid encoding the murine VISTA.COMP gene was similarly generated by inserting a synthetic dsDNA coding for the extracellular domain of VISTA, flanked by $5^{\prime}$ and $3^{\prime}$ EcoRI restriction digestion sites, upstream of the COMP pentamerization domain (residues 28-72) followed by a C-terminal hexahistidine tag. An expression plasmid coding for COMP domain alone (control) was constructed by excising the VISTA extracellular domain region from the VISTA.COMP plasmid by EcoRI restriction digestion. All plasmids encoded a $5^{\prime}$ Ig- $\kappa$ leader sequence for high protein secretion in mammalian cells. Recombinant proteins were expressed using the Expi-293 transient expression system (Thermo Fisher Scientific). Secreted VISTA-Fc was purified from culture media using HiTrap Protein A HP columns (GE Healthcare), while the histidine-tagged VISTA.COMP and COMP were purified using Ni-NTA resin (Qiagen) and desalted into PBS pH 7.4 using PD-10 columns (GE Healthcare). Proteins were verified for purity using SDS-PAGE, and protein concentration was quantified by BCA assay (Pierce) or A280 measurements.

Antibodies and ELISA kits. FACS antibodies, PE-anti-hIgG (HP6017), APC-anti-IL-2 (JES6-5H4), and PE-streptavidin were purchased from BioLegend. Anti-CD3e (145-2C11) was purchased from BioXcell. Antiphosphotyrosine (4G10) was purchased from EMD Millipore. ELISA duoset antibody pairs for IL-2, IFN- $\gamma$, IL- 6 , and TNF- $\alpha$ were purchased from R\&D Systems.

Animals. C57BL/6 mice, 8-12 weeks of age (The Jackson Laboratory), used throughout this study were housed in a pathogen-free environment at the Sunnybrook Research Institute Comparative Research facility.

Cell culture. $\mathrm{CD}^{+} \mathrm{T}$ cells were isolated from spleens of C57BL/ 6 mice using an EasySep Mouse CD4 ${ }^{+}$ $\mathrm{T}$ cell isolation kit (Stem Cell) and cultured in RPMI-1640 media supplemented with $10 \% \mathrm{FBS}, 100 \mathrm{U} / \mathrm{ml}$ penicillin, $100 \mu \mathrm{g} / \mathrm{ml}$ streptomycin, and $0.05 \mathrm{mM}$ 2-mercaptoethanol. The murine $2.10 \mathrm{~T}$ cell clone was cultured in complete IMDM supplemented with $3.5 \mathrm{ng} / \mathrm{ml} \mathrm{IL-2,} 20 \mu \mathrm{g} / \mathrm{ml}$ lecithin, and $0.5 \mathrm{mg} / \mathrm{ml} \mathrm{BSA}$

2.10 T cell clone activation. 96-well microtiter plates were coated with anti-CD3 antibody ( $3 \mu \mathrm{g} / \mathrm{ml}$ in PBS) at $4^{\circ} \mathrm{C}$ overnight. To monitor the effects of immobilized checkpoint ligands on 2.10 cell activation, the antiCD3-coated wells were washed and coated with VISTA.COMP or other recombinant proteins for 1 hour at $37^{\circ} \mathrm{C}$ in PBS. Wells were then washed with PBS (3 times) to remove residual unbound proteins. $2.10 \mathrm{~T}$ cells grown in culture were recovered, washed in IMDM (3 times), and dispensed into protein-coated wells $\left(1 \times 10^{4}\right.$ cells/well). To measure proliferation, cells were pulsed for 6 hours with $1 \mu \mathrm{Ci}[3 \mathrm{H}]$-thymidine after 18 hours of culture, and uptake was quantified using a TopCount NXT scintillation counter (Perkin Elmer). To assay the sensitivity of $2.10 \mathrm{~T}$ cells to soluble checkpoint ligands, indicated recombinant proteins were diluted in culture media and added to anti-CD3 antibody-coated wells simultaneously with the 2.10 cells

$C D 4^{+} T$ cell proliferation and cytokine secretion. Isolated murine CD4 ${ }^{+} \mathrm{T}$ cells were labeled with CFSE following the manufacturer's protocol (Thermo Fisher Scientific) and stimulated in 96-well microtiter plates precoated with an anti-CD3 antibody in the presence of murine VISTA-Fc, VISTA.COMP, or COMP alone (coated or soluble). Cells were harvested 48 or 72 hours later, and CFSE-dilution profiles were quantified by flow cytometry (FACSCalibur, Becton Dickinson). Culture media were harvested from stimulated $\mathrm{CD}^{+} \mathrm{T}$ cells at 48 or 72 hours and analyzed by ELISA to quantify VISTA.COMP-mediated inhibition of IL-2 and IFN- $\gamma$ secretion.

Flow cytometry. Binding of VISTA.COMP, VISTA-Fc, or control proteins to T cells was assessed using flow cytometry. Proteins were first biotinylated using EZ-Link Sulfo-NHS-LC-Biotin reagent (Thermo Scientific) as directed by the manufacturer. Upon completion of the reaction, the excess biotin was removed using a PD-10 (GE Healthcare) desalting column. To confirm equivalent levels of biotinylation of each protein, the quantity of biotin conjugated to each ligand was determined using HABA/Avidin reagent (Sigma-Aldrich). $2.10 \mathrm{~T}$ cells were incubated with the indicated biotinylated protein $(500 \mathrm{nM})$ or VISTA-Fc for 0.5 hours at $4^{\circ} \mathrm{C}$ in FACS staining buffer (PBS supplemented with $1 \%$ FBS and $0.09 \% \mathrm{NaN}_{3}$ ). After removal of nonbound proteins, cells were incubated with streptavidin-PE (1:300) or PE-anti-human IgG (1:100) in FACS staining buffer for 15 minutes, and the PE-fluorescence signal was analyzed using a FACSCalibur cell analyzer (Becton Dickinson). For competition experiments, VISTA-Fc and unlabeled VISTA. COMP were added to 2.10 cells at equimolar concentrations ( $500 \mathrm{nM})$, and bound VISTA-Fc was detected using PE anti-human IgG. 
Allogeneic mouse mixed leukocyte culture assay. VISTA.COMP or CD200Fc (positive control) were added to allogeneic murine mixed leukocyte cultures for 5 days, and induction of CTLs was assayed as previously described (23). Briefly, C57BL/6 responder splenocytes were incubated with an equal number of irradiated BALB/c stimulator cells in the presence of each recombinant protein at the indicated concentration. Induced CTLs were assayed by monitoring the release of ${ }^{51} \mathrm{Cr}$ from loaded P815 mastocytoma target cells over 5 hours (25:1 effector/target ratio).

Allogeneic skin graft transplant. The immunosuppressive effect of VISTA.COMP was tested in vivo using a mouse skin allograft model as previously described (23). BALB/C mice received C57BL/6 skin grafts (day 0 ) followed by treatment with VISTA.COMP (15 $\mu$ g i.v.) once every 3 days for a total of 5 treatments in combination with low-dose rapamycin $(0.5 \mathrm{mg} / \mathrm{kg}$, i.p. injections every 48 hours). A blinded investigator monitored graft survival daily.

ConA-induced acute hepatitis. The ability of VISTA.COMP to rescue mice from lethal acute inflammation was evaluated using the ConA model of acute hepatitis. Male C57BL/6 mice were treated i.p. with VISTA.COMP (200 $\mu \mathrm{g})$, VISTA-Fc, or PBS as indicated, 2 hours prior to i.v. injection of a lethal dose (15 $\mathrm{mg} / \mathrm{kg}$ ) of ConA (Sigma-Aldrich). Animals were carefully monitored and considered nonresponders and humanely sacrificed when deemed moribund (displaying excessive lethargy and unresponsiveness) by a blinded investigator. A subset of animals was sacrificed after 3 hours to quantify serum IL- 6 and TNF- $\alpha$ levels by ELISA, and the remaining animals were monitored for survival over the course of 24 hours.

Statistics. Statistical analyses were performed using GraphPad Prism software (v6.0.2) using 2-tailed Student's $t$ test unless otherwise stated. A $P$ value of less than 0.05 was considered significant. Graphs and images were created using GraphPad Prism software.

Study approval. All animal experiments and protocols were approved by the Sunnybrook Research Institute Comparative Research Animal Care Committee, accredited by the Canadian Council of Animal Care.

\section{Author contributions}

AP and JG conceptualized the study, designed all experiments, reviewed all data, and wrote the manuscript. AP, AAW, AS, NWF, MC, NC, MA, and RMG performed experimental studies. AF, NV, and MJ provided critical guidance for experimental studies. All authors reviewed and approved the final manuscript.

\section{Acknowledgments}

This work was financially supported by an operating grant from the Canadian Institute of Health Research to JG (PJT 148556). AP is the recipient of a Canada Breast Cancer Foundation fellowship.

Address correspondence to: Jean Gariépy, Department of Physical Sciences, Sunnybrook Research Institute, 2075 Bayview Avenue M7-436, Toronto, Ontario, Canada, M4N 3M5. Phone: 416.480.5710; Email: gariepy@sri.utoronto.ca.

1. Ceeraz S, Nowak EC, Noelle RJ. B7 family checkpoint regulators in immune regulation and disease. Trends Immunol. 2013;34(11):556-563.

2. Freeman GJ, et al. Engagement of the PD-1 immunoinhibitory receptor by a novel B7 family member leads to negative regulation of lymphocyte activation. J Exp Med. 2000;192(7):1027-1034.

3. Hodi FS, et al. Combined nivolumab and ipilimumab versus ipilimumab alone in patients with advanced melanoma: 2-year overall survival outcomes in a multicentre, randomised, controlled, phase 2 trial. Lancet Oncol. 2016;17(11):1558-1568.

4. Thompson CB, Allison JP. The emerging role of CTLA-4 as an immune attenuator. Immunity. 1997;7(4):445-450.

5. Topalian SL, et al. Safety, activity, and immune correlates of anti-PD-1 antibody in cancer. N Engl J Med. 2012;366(26):2443-2454.

6. Hodi FS, et al. Improved survival with ipilimumab in patients with metastatic melanoma. N Engl J Med. 2010;363(8):711-723.

7. Francisco LM, Sage PT, Sharpe AH. The PD-1 pathway in tolerance and autoimmunity. Immunol Rev. 2010;236:219-242.

8. Raptopoulou AP, et al. The programmed death 1 /programmed death ligand 1 inhibitory pathway is up-regulated in rheumatoid synovium and regulates peripheral T cell responses in human and murine arthritis. Arthritis Rheum. 2010;62(7):1870-1880.

9. Riella LV, Paterson AM, Sharpe AH, Chandraker A. Role of the PD-1 pathway in the immune response. Am J Transplant. 2012;12(10):2575-2587.

10. Lines JL, et al. VISTA is an immune checkpoint molecule for human T cells. Cancer Res. 2014;74(7):1924-1932.

11. Wang L, et al. VISTA, a novel mouse Ig superfamily ligand that negatively regulates T cell responses. J Exp Med. 2011;208(3):577-592.

12. Flies DB, et al. Coinhibitory receptor PD-1H preferentially suppresses CD4+ T cell-mediated immunity. J Clin Invest. 2014;124(5):1966-1975.

13. Lines JL, Sempere LF, Broughton T, Wang L, Noelle R. VISTA is a novel broad-spectrum negative checkpoint regulator for 
cancer immunotherapy. Cancer Immunol Res. 2014;2(6):510-517.

14. Le Mercier I, et al. VISTA regulates the development of protective antitumor immunity. Cancer Res. 2014;74(7):1933-1944.

15. Ceeraz S, et al. VISTA deficiency accelerates the development of fatal murine lupus nephritis. Arthritis Rheumatol. 2017; 69(4):814-825.

16. Wang L, et al. Disruption of the immune-checkpoint VISTA gene imparts a proinflammatory phenotype with predisposition to the development of autoimmunity. Proc Natl Acad Sci USA. 2014;111(41):14846-14851.

17. Li N, et al. Immune-checkpoint protein VISTA critically regulates the IL-23/IL-17 inflammatory axis. Sci Rep. 2017;7(1):1485.

18. Yoon KW, et al. Control of signaling-mediated clearance of apoptotic cells by the tumor suppressor p53. Science. 2015;349(6247):1261669.

19. Flies DB, Higuchi T, Chen L. Mechanistic assessment of PD-1H coinhibitory receptor-induced T cell tolerance to allogeneic antigens. J Immunol. 2015;194(11):5294-5304.

20. Cho CH, et al. COMP-Ang1: a designed angiopoietin-1 variant with nonleaky angiogenic activity. Proc Natl Acad Sci USA. 2004;101(15):5547-5552.

21. Efimov VP, Lustig A, Engel J. The thrombospondin-like chains of cartilage oligomeric matrix protein are assembled by a five-stranded alpha-helical bundle between residues 20 and 83. FEBS Lett. 1994;341(1):54-58.

22. Haughn L, Gratton S, Caron L, Sékaly RP, Veillette A, Julius M. Association of tyrosine kinase p56lck with CD4 inhibits the induction of growth through the alpha beta T cell receptor. Nature. 1992;358(6384):328-331.

23. Prodeus A, et al. Agonistic CD200R1 DNA aptamers are potent immunosuppressants that prolong allogeneic skin graft survival. Mol Ther Nucleic Acids. 2014;3:e190.

24. Tiegs G, Hentschel J, Wendel A. A T cell-dependent experimental liver injury in mice inducible by concanavalin A. J Clin Invest. 1992;90(1):196-203.

25. Lin DY, et al. The PD-1/PD-L1 complex resembles the antigen-binding Fv domains of antibodies and T cell receptors. Proc Natl Acad Sci USA. 2008;105(8):3011-3016.

26. van der Merwe PA, Bodian DL, Daenke S, Linsley P, Davis SJ. CD80 (B7-1) binds both CD28 and CTLA-4 with a low affinity and very fast kinetics. J Exp Med. 1997;185(3):393-403.

27. Nowak EC, et al. Immunoregulatory functions of VISTA. Immunol Rev. 2017;276(1):66-79.

28. Yang W, et al. Construction of a versatile expression library for all human single-pass transmembrane proteins for receptor pairings by high throughput screening. J Biotechnol. 2017;260:18-30. 